\title{
Disruption of the surfactant protein A receptor SP-R210L (CD245 $\alpha / M Y 018 A \alpha)$ alters respiratory function and iron sequestration in alveolar macrophages of aged mice
}

Eric Yau ${ }^{1}$, Todd M Umstead ${ }^{1}$, Raz Abdulqadir ${ }^{1}$, Kristin Fino¹, Zhiwei Guan ${ }^{1}$, Sanmei Hu${ }^{1}$, Susan DiAngelo', Kavitha Hassan¹, Sarah S. Bingaman², Hannah Atkins ${ }^{3}$, Timothy K Cooper $^{3}$, Amy C. Arnold ${ }^{2}$, E. Scott Halstead ${ }^{4}$, and Zissis C. Chroneos ${ }^{1, *}$

${ }^{1}$ Department of Pediatrics, Microbiology and Immunology, Pulmonary Immunology and Physiology Laboratory, Pennsylvania State University College of Medicine, Pennsylvania, USA

${ }^{2}$ Department of Neural and Behavioral Sciences, Pennsylvania State University College of Medicine, Pennsylvania, USA

${ }^{3}$ Department of Comparative Medicine, Pennsylvania State University College of Medicine, Pennsylvania, USA

${ }^{4}$ Department of Pediatrics, Division of Pediatric Critical Care Medicine, Pulmonary Immunology and Physiology Laboratory, Pennsylvania State University College of Medicine, Pennsylvania, USA

Correspondence:

Zissis C. Chroneos, Ph.D. 


\section{ABSTRACT}

Previous studies demonstrated that the host defense collectins, surfactant protein A and complement component 1q, modulate tissue-dependent macrophage activation, pathogen clearance, and regulatory macrophage functions through the receptor SPR210, which consists of two isoforms SP-R210L and SP-R210s. These isoforms are encoded by alternatively spliced mRNAs of the Myo18aMYO18A gene in mice and humans. The present study in conditional transgenic mice revealed novel age-related functions of the SP-R210L isoform in modulating pulmonary mechanics, iron sequestration in alveolar macrophages (AMs), and life-long maintenance of the alveolar macrophage population. Our findings support the novel idea that SP-R210L-deficient AMs undergo bi-directional epigenetic adaptation that results in chronic dysregulation of broncho-alveolar function, immune homeostasis, and maintenance of oncotic balance at the airway-capillary interface. Disruption of SP-R210 increases the risk for development of severe interstitial lung disease during development and aging. 


\section{INTRODUCTION}

The macrophage-expressed isoforms of myosin XVIIIA (MYO8A $\alpha$ and MYO18A $\beta$ ), named as SP-R210 L and SP-R210s, respectively, mediate immune and host defense functions as shared receptors for surfactant protein A (SP-A) and complement component $1 \mathrm{q}(\mathrm{C} 1 \mathrm{q})$ in alveolar and peritoneal macrophages, respectively, as well as autonomous modulation of macrophage phenotype (1-6). SP-R210 is identical to the previously orphan antigen CD245 (7). Alternative splicing of Myo18A generates multiple protein isoforms categorized broadly as MYO18A $\alpha, \mathrm{MYO18A} \beta$, and MYO18A $\gamma$. MYO18A $\alpha$ and MYO18A $\gamma$ are distinguished from MYO18A $\beta$ by different amino-terminal PDZ domain and polyproline extensions, respectively and an additional carboxy-terminal tail in MYO18A $\gamma$ (8). Myo18A $\alpha$ is more widely expressed, although not in the liver, heart, or skeletal muscle (9). MYO18A $\beta$ is found in hematopoietic tissues, whereas MYO18A $\gamma$ is exclusive to heart and skeletal muscle (8-10). SP-R210L occurs in mature macrophages, whereas SPR210s occurs in both mature and immature myeloid cells $(9,10)$. SP-A expression outside the lung and during pregnancy mediates immunological surveillance and physiological control of uterine smooth muscle and myometrial cell contractility, smooth muscle phenotype and vascular remodeling, and ocular neovascularization in the neonate (1116). To discern the multifaceted SP-A-dependent and independent functions of SP-R210, we studied the basal phenotype of transgenic mice with conditional deletion of the SPR210L isoform. Our findings support the model that SP-R210L modulates bi-directional communication between AMs and epithelial and vascular cells in the lung. 


\section{RESULTS AND DISCUSSION}

Selective deletion of SP-R210L was achieved by Cre-mediated inversion recombination of a knock-in exon 1 allele with flanking Lox66/Lox77 recombination sites (Figure 1A) $(17,18)$. Breeding of $S P-R 210_{L}^{f l /+}$ with Itgax (CD11C)Cre mice (19) disrupted SP-R210L but not SP-R210s expression in AMs SP-R210L Invfl/t (Figure 1B-E). Exon 1 encodes the KE and PDZ domains of MYO18A $\alpha$. Itgax ${ }^{\text {Cre }}$ targets mainly CD11 $c^{\text {hi }}$ AMs and dendritic cells (19). Immunofluorescence microscopy staining of lung tissue sections from $S P-R 21 L_{L}^{f /+}$ littermate control mice(Figure 1F) and SP-R210L-deficient lungs (Figure 1H) localized SP-A to alveolar type II epithelial cells (yellow arrows), alveolar lumen, and bronchial club cells (white arrows) as previously established (20). SP-R210, however, localized to thin alveolar septa and the apical surface of dome shaped club cells in both control and SP-R210L-deficient lungs (Figure 1F-G, white arrows). SP-R210L is the main isoform found in lung tissue and in stoichiometric expression with SP-R210s in AMs (Figure 1C)(9). The overall fluorescence intensity for SP-R210 decreased by $20 \%$ in SPR210L-deficient lungs compared to littermates, whereas SP-A staining was similar (Figure $1 \mathrm{H})$. The number of AMs in aged SP-R210L-deficient mice decreased compared to littermates (Figure 1J), indicating AM attrition. The lung histology of SP-R210L-deficient mice did not show discernible abnormalities other than lymphocytic aggregates compared to littermates (Figure 1J,M). The SP-R210L-deficient AMs, however, stained positive for hemosiderin (Figure $1 \mathrm{~N}-\mathrm{O}$ ) compared to no staining in controls (Figure $1 \mathrm{~K}-\mathrm{L}$ ). Hemosiderin was confirmed in isolated SP-R210L-deficient AMs (Figure 1P-R), with highest levels in homozygous SP-R210L-deficient AMs (Figure 1S). BAL extracellular iron levels were not different (Figure 1T). 
Macrophage hemosiderin formation is part of normal iron flux and not readily detectable $(21,22)$. Under pathological conditions, AM hemosiderin develops from incomplete lysosomal degradation of ferritin, heme iron proteins, and partial hemoglobin degradation following erythrophagocytosis (23). SP-R210L-deficient mice did not develop abnormalities in systemic erythrocyte metabolism as indicated by similar hemosiderin spleen deposition (Figure 2A), hemoglobin degradation (Figure 2B), and kidney function (Figure 2C-D). Blood urea nitrogen levels (BUN) were similar at 2-months of age, although lower than controls in 6-months-old SP-R210L-deficient mice (Figure 2D). The aged SP-R210L-deficient mice did not develop tachypnea or dyspnea (Figure 2E). Basal capillary permeability was not affected (Figure $2 \mathrm{~F}$ ) as indicated by similar extravasation of Evans blue in the lung, although the circulating Evans blue concentration in SP-R210Ldeficient mouse blood was $10 \%$ lower than littermates (Figure $2 \mathrm{G}$ ), suggesting increased capillary volume. The lungs of 6-month-old SP-R210L-deficient mice, however, retained more fluid during heart perfusion compared to littermates (Figure $2 \mathrm{H}$ ), indicating increased water permeability at increased pressure.

Adding to this underlying pathophysiology, a 6-week-old female mouse presenting with lethargy and anorexia suffered from severe cardiopulmonary disease and otitis media (Figure 2I-N). Histological evaluation showed right ventricular and asymmetric septal hypertrophy (Figure 2I), unilateral otitis media with intraluminal serous exudate and neutrophilic inflammation affecting mainly the middle ear, and fibroplasia thickening of the ear canal lining (Figure 2J). In this regard, disruption of TGIF an inhibitor of TGF $\beta$ activation, a known effector of SP-R210 (24), leads to chronic otitis media (25). This case coincided with the presence of Klebsiella oxytoca and Pseudomonas 
aeruginosa in the mouse colony, suggesting an infectious trigger. Gross evaluation showed parenchymal lung hemorrhage (Figure 2K). Histopathological evaluation showed multifocal to coalescing expansion of alveolar septa with abundant nascent and mature fibrosis, abundance of foamy and frequently hemosiderin-laden intra-alveolar macrophages and fibrous projections, eosinophilic debris, and acute hemorrhage (Figure 2L-M). Mason's trichrome staining showed fibrotic thickening of alveolar septa (Figure 2N). Small and medium arteries were dilated, tortuous, and redundant, with smooth muscle hyperplasia in smaller arteries (Figure 2L-N).

Comparative transcriptome profile analysis between SP-R210L-deficient and littermate AMs (Figure 2O-P) showed moderate increase in hemoglobin mRNAs, Hba1, $\mathrm{Hba2}$, and $\mathrm{Hbb}$, supporting erythrophagocytosis as the source of hemosiderin (Figure 2O) (26). Relevant to iron metabolism, RNAsequencing showed moderate decreases in heme and iron transport protein mRNAs for Hrg1 (Slc48a1) (27), ceruloplasmin (Cp) (28), Steap4 (29), haptoglobin (Hp) (30), and lipocalin 2 (Lcn2) (31), indicating reduced iron import and intrinsic iron flux in SP-R210L-deficient AMs, responses consistent with iron abundance. The iron and $\alpha$-ketoglutarate-dependent histone lysine demethylase $K d m 4 b$ gene (32) was the most highly expressed mRNA in SP-R210L-deficient AMs (Figure 10), indicating epigenetic adaptation. KDM4B modulates repressive and non-repressive chromatin balance (32). One noteworthy KDM4B effector is PPAR $\gamma$, which is central to surfactant metabolism in AMs (33). Relevant triggers of KDM4B expression include hypoxia (32) and osmolarity (34), conditions encountered during gestation and transition from the hypoxia in utero to normoxia in postnatal life (35-39), and TGF $\beta$ (32). In the 
present context, AMs may sense changes in oncotic balance in the lung's airway-capillary barrier.

Upstream pathway analysis of mRNA sequencing data revealed reciprocal inhibition of inflammatory (Tnfa, II1 $\beta$, RelA, Myd88, II-17a, Ifn $\gamma$ ) and activation of antiinflammatory pathways (Cish, Zfp36, II-10ra, Gfi1, Dusp1, II37, Siggir) in SP-R210Ldeficient AMs (Figure 2P). Suppression of the Csf2 signaling pathway (Figure 2P-Q) predicts decreased responsiveness of SP-R210L-deficient AMs to GM-CSF. Among the upregulated pathways, the IL-37/SIGGIR pathway (Figure 2P) can antagonize GM-CSF signaling (40). Relevant to the present findings, IL-37 is a proangiogenic (41) cytokine produced primarily by monocytes and dendritic cells (42). Furthermore, GM-CSF antagonizes SP-A binding and alters SP-A binding behavior in a concentration-dependent manner $(43,44)$. Conversely, SP-A maintains stoichiometric balance of SP-R210L and SP-R210s on AMs (2), modulates GM-CSF secretion (45), and has increased potency at suppressing TNF $\alpha$ in SP-R210L-deficient macrophages (2). The reduction in Csf2 was associated with decreased AM number in both 4-month-old (Figure 2R) and aged SPR210L-deficient mice compared to littermates (Figure 1 l above). The number of lung CD45+ cells and monocytes were also suppressed (Figure 2S). To this end, monocytic recruitment genes (e.g. Sell, S100A8/9, Cxcr2, TNF $\alpha$ ) were decreased (Figure 2O). Furthermore, canonical pathway analysis showed reduction in adhesion and diapedesis pathways in SP-R210L-deficient AMs (Figure 2T). Conversely, reduction in $\alpha$-adrenergic receptor signaling (Figure 2S), suggested decline in neuro-hormonal control of SP-R210Ldeficient AMs. In this context, cardiovascular autonomic (Figure 3A-H), arterial oxygen 
(Figure 3I), and hematological measurements (Figure 3J-P) did not support development of cardiac dysfunction in aging SP-R210L-deficient mice.

We next assessed if SP-R210L deletion alters respiratory mechanics. Pressurevolume curves showed that the lungs of 6-month-old SP-R210L-deficient mice were less distensible (Figure 4A) and had increased expiratory curve slope (Figure 4B), indicating development of chronic lung disease. The age-associated increase in Inspiratory capacity (Figure 4C) and compliance (Figure 4D) in SP-R210L-deficient mice was slower than in littermates. Respiratory system (Figure 2E) and tissue elastance (Figure 2F) were not different. Central airway Newtonian resistance, however, increased over time in SPR210L-deficient mice (Figure 4G), whereas tissue damping was not affected (Figure 4I). Respiratory system resistance remained stable in SP-R210L-deficient mice compared to decline in littermates (Figure 4I). All age-related changes in respiratory mechanics measured in control littermates reproduce previous studies in WT mice (46). Methacholine challenge studies showed enhanced airway responsiveness for respiratory system and tissue elastance (Figure 4J-K) and Newtonian resistance (Figure 4L) in 6month-old SP-R210L-deficient mice compared to littermates. Tissue damping (Figure 4M) and respiratory system resistance (Figure $4 \mathrm{~N}$ ) where not different.

Taken together, our findings indicate that SP-R210 isoforms modulate intercellular communication in the lung. Transcriptome data support the model that SP-R210 isoforms modulate rheostatic functions of SP-A and GM-CSF in the lung through epigenetic adaptation to local conditions. Selective disruption of the SP-R210 isoform resulted in subclinical disease presenting as hemosiderin deposition, attrition of AMs, and changes in airway-capillary mechanics and basal fluid homeostasis in the lung. Rheological 
adaptation as indicated by differences in blood urea, serum Evans blue dye concentration, and hydrostatic fluid retention in older SP-R210L-deficient mice suggests compensatory oncotic fluid distribution to maintain respiratory function. Our study raises new questions about the mechanisms by which SP-R210 isoforms regulate immune and surfactant homeostasis, host defense, and respiratory physiology during development and aging.

\section{METHODS}

The conditional Myo18 $\alpha$ knock-in mice, designated as $S P-R 21 L_{L}^{f / /+}$, were generated by Ozgene Pty Ltd. (Bentley WA, Australia) on a C57BL/6 background by homologous recombination. A knock-in exon 1 allele flanked by Lox66/Lox77 inversion recombination sites was made using standard molecular cloning techniques and transfected by electroporation in C57BL/6 embryonic stem cells (ES) cells. Homologous recombinant ES cells identified by Southern blotting were microinjected into C57BL/6 blastocysts. Founder transgenic were bred to establish the SP-R210 $L^{\text {flox/++}}$ mouse colony and then with Itgax Cre mice for conditional deletion of SP-R210L as detailed in "Supplemental Material and Methods". All procedures were approved by the Institutional Animal Care and Use Committee of Pennsylvania State University College of Medicine and adhered to the $8^{\text {th }}$ edition of the PHS NIH GUIDE for the care and use of laboratory animals. 


\section{AUTHOR CONTRIBUTIONS}

EY, TMU, RA, KF, ZG, SH, SD, KH, and SB conducted experiments, acquired and graphed data, and edited the manuscript; EY processed and analyzed genomic data; HA and TKC conducted pathological diagnosis and blind scoring of histopathology; ACA and ESH designed cardiovascular and flow cytometry experiments, analyzed data and edited the manuscript; ZCC led and designed the study, analyzed data, and wrote the manuscript

\section{ACKNOWLEDGEMENTS}

This work was funded by PHS grants HL128746, Pennsylvania Department of Health, The Children's Miracle Network, and the Department of Pediatrics Pennsylvania State University College of Medicine. We would like to thank Nate Schaffer and Joseph Bednarzyk from the Penn State College of Medicine Flow Cytometry Core Facility as well as the Institute of Personalized Medicine for assistance with genomic processes.

\section{CONFLICTS OF INTEREST}

Zissis C. Chroneos is co-founder of Respana Therapeutic, Inc. (http://respanatherapeutics.com/) an early-stage company developing therapeutics targeting SP-R210 isoforms and is co-inventor on associated patents. 


\section{FIGURE LEGENDS}

Figure 1. Disruption of SP-R210L results in hemosiderin deposition in AMs and decline in AM number. A-B) Schematic depiction and targeting of the floxed Myo18A $\alpha$ exon 1 by Cre-mediated inversion recombination. The targeted SPR210 IInvfl/+ allele was identified by Southern blot hybridization (B) and selective deletion of SP-R210เ protein was demonstrated by Western blotting (C) and densitometry analysis (D-E). D-E $n=4$ mice per group. ${ }^{* *} p<0.001$. F-G) Immunofluorescence microscopy and imaging of lung tissue sections showed similar distribution of SP-A and SP-R210 in control (F) and SP-R210Ldeficient lungs $(G)$ and partial reduction in SP-R210 fluorescence intensity $(H)$ of SPR210 in SP-R210L-deficient lungs compared to littermates. I) Flow cytometry analysis showed reduction CD45+ cells and CD11 b ${ }^{\text {lo }}$ AMs cell number in BAL from aged 9-12month old SP-R210L-deficient mice. J-O) H\&E staining showed similar airway and alveolar histology of littermate (J) and SP-R210L-deficient lungs (M) and bronchocentric lymphocytic aggregates in the latter (yellow star in M). Perl's Prussian blue staining revealed hemosiderin iron in SP-R210L-deficient AMs (N-O) but not littermate AMs (K-L). Perl's iron staining of isolated AMs (P-R) confirmed lack of hemosiderin staining in littermate AMs (P) compared to hemosiderin AMs from heterozygous (Q) and homozygous (R) SP-R210L-deficient mice. S) Golde scoring showed proportional increase in hemosiderin ladden AMs in heterozygous and homozygous SP-R210Ldeficient mice. For Golde scoring, $\mathrm{n}=8-12$ mice per group at 8-11-months of age. ${ }^{* * * *} p<0.0001$. K) Similar concentration of free iron in BAL control and SP-R210L-deficient AMs. 
Figure 2. Disruption of SP-R210L alters lung hydrostatic solute distribution, AM transcriptome phenotype, basal extravasation of monocyte subsets in the lung, and is associated with rare cardiopulmonary interstitial disease. Histological scoring (A) and blood measurements (B-D) showed similar hemosiderin deposition in spleens of 2- and 6-momth old control and heterozygous SP-R210L-deficient mice as well as similar levels of T-bilirubin (B) and creatinine (C) in blood, whereas blood urea nitrogen (D) did not increase with age in SP-R210L-deficient mice. $\mathrm{N}=4$ mice per group ${ }^{*} \mathrm{p}<0.05$. Respiratory rates were also not different (E). F-H) Evans blue (EB) dye permeability experiments showed similar lung microvascular permeability of EB in 6-month old SPR210L-deficient mice compared to littermates $(F)$, whereas the concentration of circulating EB was lower $\left(n=4,{ }^{*} p<0.05\right)$. Perfused SP-R210L-deficient lungs retained more fluid compared to littermates ( $n=6$ per genotype in the unperfused group, and $n=8$ mice per genotype in the perfused group $\left.{ }^{*}<0.05,{ }^{* *} p<0.001\right)$. I-N) Development of pulmonary hypertension (I), otitis media $(\mathrm{J})$, lung hemorrhage $(\mathrm{K})$, and intra-alveolar and interstitial fibrosis vasculopathy in a SP-R210L-deficient mouse (L-N) as detailed in "Results and Discussion". (O-Q) Differential transcriptome profile $(\mathrm{O})$ and upstream pathway analysis of SP-R210L-deficient AMs vs littermates (P-Q) indicate decreased threshold of basal AM activation. (R-S) Flow cytometry analysis showed decreased number of CD45+ cells and CD11 b $\mathrm{b}^{\circ} \mathrm{AMs}$ in BAL $(\mathrm{R})$ and decreased number of both $\mathrm{CD} 45+$ immune cells and monocyte subsets (S) in lungs of 4-month old SP-R210L-deficient mice compared to littermates. (R-S, $\mathrm{n}=6$ littermates and $\mathrm{n}=12$ for Itgax ${ }^{C r e} S P R 21 \mathrm{~L}^{\text {Invt// }}, \mathrm{p}<0.05,{ }^{* * *} \mathrm{p}<0.001$ ) 
Figure 3. Disruption of SP-R210 L does not alter cardiac function, respiratory gas exchange, or hematologic parameters during aging. Blood pressure $(A-C)$, heart rate (D) and cardiac autonomic tone (E-G) were obtained under isoflurane anesthesia $(n=6$ mice/group at 2- and 6-months of age, and $n=4 /$ group at 8 -months of age). Heart rate $(H)$ and arterial oxygen saturation (I) in aged 13-14-month-old littermate $(n=10)$, heterozygous ( $n=3)$, and homozygous ( $n=8)$ SP-R210L-deficient mice were measured using a MouseOx pulse oximeter under ketamine/xylazine anesthesia. Hematological parameters $(\mathrm{J}-\mathrm{O})$ and serum chemistry $(\mathrm{P})$ were determined in blood collected via the cauda vena cava under ketamine/xylazine anesthesia. $\mathrm{N}=4$ mice/group. All methods are described in "Supplemental Materials and Methods" and results detailed in "Results and Discussion".

Figure 4. Disruption of SP-R210L results in age-dependent alterations in basal respiratory mechanics and airway hyper responsiveness. Respiratory mechanics parameters were obtained using the forced oscillation technique as detailed in "Supplemental Materials and Methods" and "Results and Discussion" sections. N=4 mice per genotype per time point. ${ }^{*} p<0.05,{ }^{* \star *} p<0.001$. 


\section{REFERENCES}

1. Minutti CM, Jackson-Jones LH, Garcia-Fojeda B, Knipper JA, Sutherland TE, Logan N, et al. Local amplifiers of IL-4Ralpha-mediated macrophage activation promote repair in lung and liver. Science. 2017;356(6342):1076-80.

2. Yang L, Carrillo M, Wu YM, DiAngelo SL, Silveyra P, Umstead TM, et al. SP-R210 (Myo18A) Isoforms as Intrinsic Modulators of Macrophage Priming and Activation. PLoS One. 2015;10(5):e0126576.

3. Sever-Chroneos Z, Krupa A, Davis J, Hasan M, Yang CH, Szeliga J, et al. Surfactant protein A (SP-A)-mediated clearance of Staphylococcus aureus involves binding of SP-A to the staphylococcal adhesin eap and the macrophage receptors SP-A receptor 210 and scavenger receptor class A. J Biol Chem. 2011;286(6):4854-70.

4. Chroneos ZC, Sever-Chroneos Z, and Shepherd VL. Pulmonary surfactant: an immunological perspective. Cell Physiol Biochem. 2010;25(1):13-26.

5. Chroneos ZC, Abdolrasulnia R, Whitsett JA, Rice WR, and Shepherd VL. Purification of a cell-surface receptor for surfactant protein A. J Biol Chem. 1996;271(27):16375-83.

6. Casals C, Garcia-Fojeda B, and Minutti CM. Soluble defense collagens: Sweeping up immune threats. Mol Immunol. 2019;112:291-304.

7. De Masson A, Giustiniani J, Marie-Cardine A, Bouaziz JD, Dulphy N, Gossot D, et al. Identification of CD245 as myosin 18A, a receptor for surfactant A: A novel pathway for activating human NK lymphocytes. Oncoimmunology. 2016;5(5):e1127493.

8. Horsthemke M, Nutter LMJ, Bachg AC, Skryabin BV, Honnert U, Zobel T, et al. A novel isoform of myosin 18A (Myo18Agamma) is an essential sarcomeric protein in mouse heart. J Biol Chem. 2019;294(18):7202-18.

9. Yang $\mathrm{CH}$, Szeliga J, Jordan J, Faske S, Sever-Chroneos Z, Dorsett B, et al. Identification of the surfactant protein $A$ receptor 210 as the unconventional myosin 18A. J Biol Chem. 2005;280(41):34447-57.

10. Mori K, Furusawa T, Okubo T, Inoue T, Ikawa S, Yanai N, et al. Genome structure and differential expression of two isoforms of a novel PDZ-containing myosin (MysPDZ) (Myo18A). J Biochem. 2003;133(4):405-13.

11. Vieira F, Kung JW, and Bhatti F. Structure, genetics and function of the pulmonary associated surfactant proteins $A$ and $D$ : The extra-pulmonary role of these $C$ type lectins. Ann Anat. 2017;211:184-201.

12. Ujma S, Horsnell WG, Katz AA, Clark HW, and Schafer G. Non-Pulmonary Immune Functions of Surfactant Proteins A and D. J Innate Immun. 2017;9(1):311.

13. Ran R, Cai D, King SD, Que X, Bath JM, and Chen SY. Surfactant Protein A, a Novel Regulator for Smooth Muscle Phenotypic Modulation and Vascular Remodeling. Arterioscler Thromb Vasc Biol. 2020:ATVBAHA120314622.

14. Condon JC, Jeyasuria P, Faust JM, and Mendelson CR. Surfactant protein secreted by the maturing mouse fetal lung acts as a hormone that signals the initiation of parturition. Proc Natl Acad Sci U S A. 2004;101(14):4978-83. 
15. Sotiriadis G, Dodagatta-Marri E, Kouser L, Alhamlan FS, Kishore U, and Karteris E. Surfactant Proteins SP-A and SP-D Modulate Uterine Contractile Events in ULTR Myometrial Cell Line. PLoS One. 2015;10(12):e0143379.

16. Reinl EL, and England SK. Fetal-to-maternal signaling to initiate parturition. J Clin Invest. 2015;125(7):2569-71.

17. Kontgen F, Suss G, Stewart C, Steinmetz M, and Bluethmann H. Targeted disruption of the MHC class II Aa gene in C57BL/6 mice. Int Immunol. 1993;5(8):957-64.

18. Kontgen $\mathrm{F}$, and Stewart CL. Simple screening procedure to detect gene targeting events in embryonic stem cells. Methods Enzymol. 1993;225:878-90.

19. Caton ML, Smith-Raska MR, and Reizis B. Notch-RBP-J signaling controls the homeostasis of CD8- dendritic cells in the spleen. J Exp Med. 2007;204(7):165364.

20. Auten RL, Watkins RH, Shapiro DL, and Horowitz S. Surfactant apoprotein A (SPA) is synthesized in airway cells. Am J Respir Cell Mol Biol. 1990;3(5):491-6.

21. Epstein CE, Elidemir O, Colasurdo GN, and Fan LL. Time course of hemosiderin production by alveolar macrophages in a murine model. Chest. 2001;120(6):201320.

22. Crichton RR, and Ward RJ. Iron homeostasis. Met lons Biol Syst. 1998;35:633-65.

23. Persson HL, and Vainikka LK. Lysosomal iron in pulmonary alveolar proteinosis: a case report. Eur Respir J. 2009;33(3):673-9.

24. Samten B, Townsend JC, Sever-Chroneos Z, Pasquinelli V, Barnes PF, and Chroneos ZC. An antibody against the surfactant protein A (SP-A)-binding domain of the SP-A receptor inhibits T cell-mediated immune responses to Mycobacterium tuberculosis. J Leukoc Biol. 2008;84(1):115-23.

25. Tateossian H, Morse S, Parker A, Mburu P, Warr N, Acevedo-Arozena A, et al. Otitis media in the Tgif knockout mouse implicates TGFbeta signalling in chronic middle ear inflammatory disease. Hum Mol Genet. 2013;22(13):2553-65.

26. Summers KM, Bush SJ, and Hume DA. Network analysis of transcriptomic diversity amongst resident tissue macrophages and dendritic cells in the mouse mononuclear phagocyte system. PLoS Biol. 2020;18(10):e3000859.

27. White C, Yuan X, Schmidt PJ, Bresciani E, Samuel TK, Campagna D, et al. HRG1 is essential for heme transport from the phagolysosome of macrophages during erythrophagocytosis. Cell Metab. 2013;17(2):261-70.

28. Yang Q, Liu W, Zhang S, and Liu S. The cardinal roles of ferroportin and its partners in controlling cellular iron in and out. Life Sci. 2020;258:118135.

29. Scarl RT, Lawrence CM, Gordon HM, and Nunemaker CS. STEAP4: its emerging role in metabolism and homeostasis of cellular iron and copper. $J$ Endocrinol. 2017;234(3):R123-R34.

30. Yang F, Friedrichs WE, Navarijo-Ashbaugh AL, deGraffenried LA, Bowman BH, and Coalson JJ. Cell type-specific and inflammatory-induced expression of haptoglobin gene in lung. Lab Invest. 1995;73(3):433-40.

31. Xiao X, Yeoh BS, and Vijay-Kumar M. Lipocalin 2: An Emerging Player in Iron Homeostasis and Inflammation. Annu Rev Nutr. 2017;37:103-30.

32. Wilson C, and Krieg AJ. KDM4B: A Nail for Every Hammer? Genes (Basel). 2019;10(2). 
33. Baker AD, Malur A, Barna BP, Ghosh S, Kavuru MS, Malur AG, et al. Targeted PPAR \{gamma\} deficiency in alveolar macrophages disrupts surfactant catabolism. Journal of lipid research. 2010;51(6):1325-31.

34. Zhang Y, Wang Y, Zhou X, Wang J, Shi M, Wang J, et al. Osmolarity controls the differentiation of adipose-derived stem cells into nucleus pulposus cells via histone demethylase KDM4B. Mol Cell Biochem. 2020;472(1-2):157-71.

35. Gao Y, Cornfield DN, Stenmark KR, Thebaud B, Abman SH, and Raj JU. Unique aspects of the developing lung circulation: structural development and regulation of vasomotor tone. Pulm Circ. 2016;6(4):407-25.

36. Mammoto A, and Mammoto T. Vascular Niche in Lung Alveolar Development, Homeostasis, and Regeneration. Front Bioeng Biotechnol. 2019;7:318.

37. Nakanishi H, Morikawa S, Kitahara S, Yoshii A, Uchiyama A, Kusuda S, et al. Morphological characterization of pulmonary microvascular disease in bronchopulmonary dysplasia caused by hyperoxia in newborn mice. Med Mol Morphol. 2018;51(3):166-75.

38. Moen V, Brudin L, Tjernberg I, Rundgren M, and Irestedt L. Feto-maternal osmotic balance at term. A prospective observational study. $J$ Perinat Med. 2018;46(2):183-9.

39. Puthiyachirakkal M, and Mhanna MJ. Pathophysiology, management, and outcome of persistent pulmonary hypertension of the newborn: a clinical review. Front Pediatr. 2013;1:23.

40. Nold-Petry CA, Lo CY, Rudloff I, Elgass KD, Li S, Gantier MP, et al. IL-37 requires the receptors IL-18Ralpha and IL-1R8 (SIGIRR) to carry out its multifaceted antiinflammatory program upon innate signal transduction. Nat Immunol. 2015;16(4):354-65.

41. Yang T, Lin Q, Zhao M, Hu Y, Yu Y, Jin J, et al. IL-37 Is a Novel Proangiogenic Factor of Developmental and Pathological Angiogenesis. Arterioscler Thromb Vasc Biol. 2015;35(12):2638-46.

42. Shibata Y, Berclaz PY, Chroneos ZC, Yoshida M, Whitsett JA, and Trapnell BC. GM-CSF regulates alveolar macrophage differentiation and innate immunity in the lung through PU.1. Immunity. 2001;15(4):557-67.

43. Yoshida M, Ikegami M, Reed JA, Chroneos ZC, and Whitsett JA. GM-CSF regulates protein and lipid catabolism by alveolar macrophages. Am J Physiol Lung Cell Mol Physiol. 2001;280(3):L379-86.

44. Chroneos Z, and Shepherd VL. Differential regulation of the mannose and SP-A receptors on macrophages. Am J Physiol. 1995;269(6 Pt 1):L721-6.

45. Blau H, Riklis S, Kravtsov V, and Kalina M. Secretion of cytokines by rat alveolar epithelial cells: possible regulatory role for SP-A. Am J Physiol. 1994;266(2 Pt 1):L148-55.

46. Veldhuizen RAW, McCaig LA, Pape C, and Gill SE. The effects of aging and exercise on lung mechanics, surfactant and alveolar macrophages. Exp Lung Res. 2019;45(5-6):113-22. 


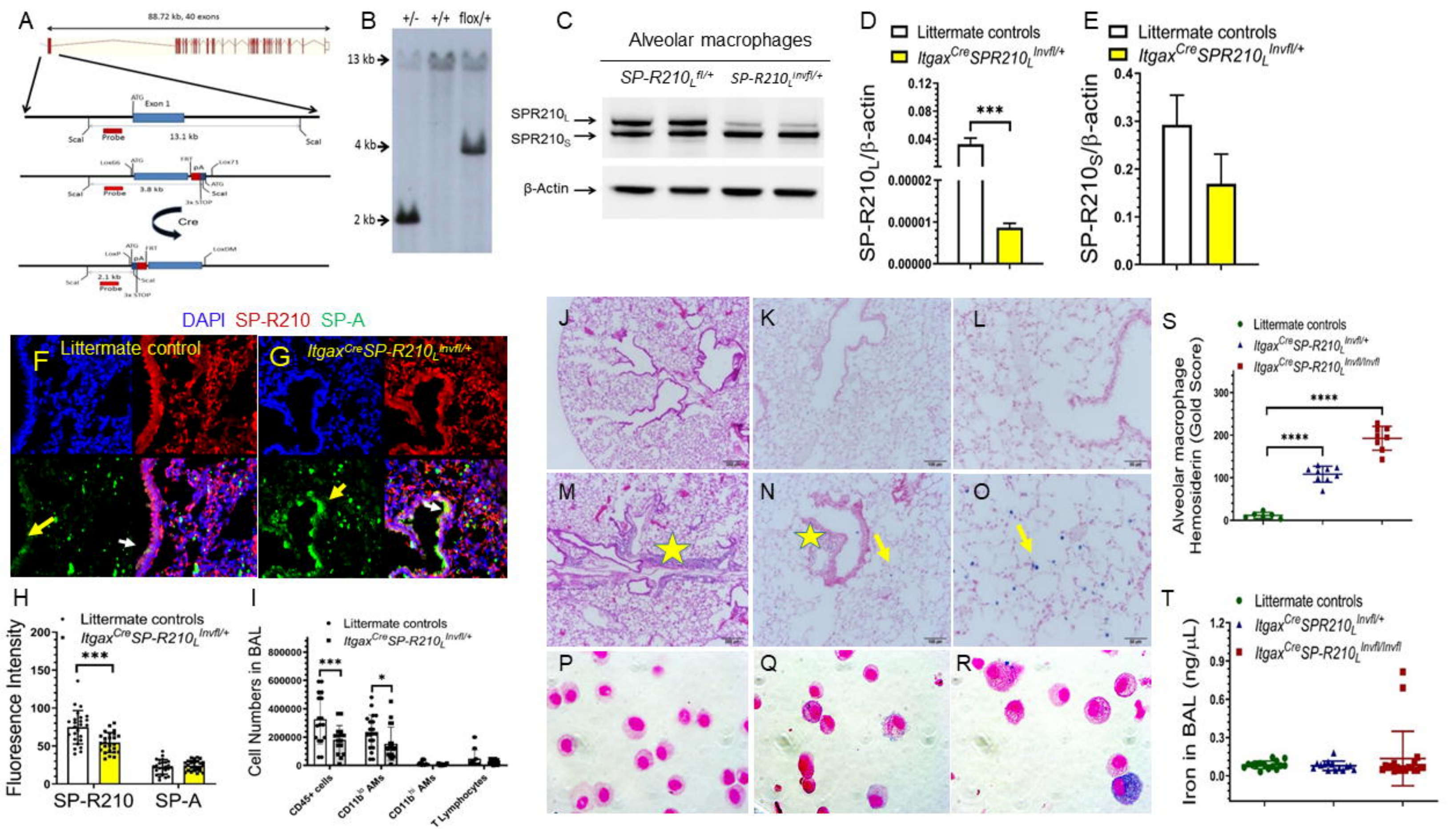



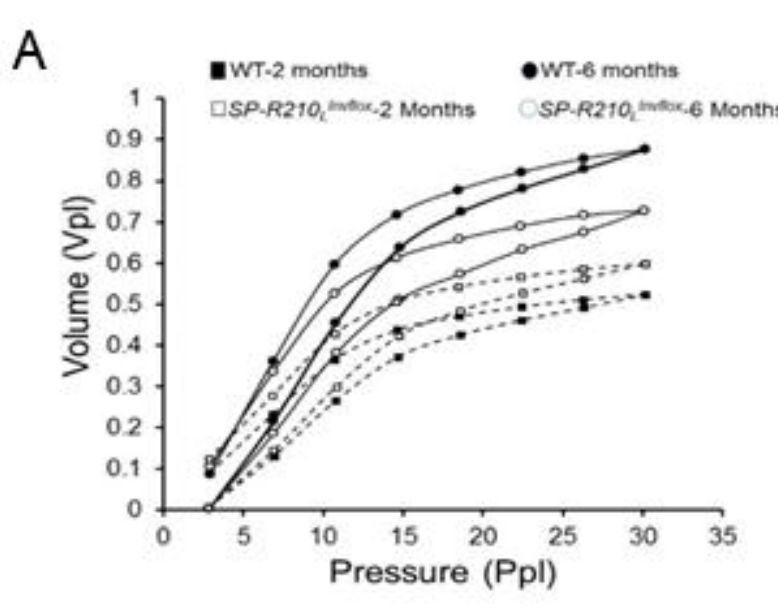

\section{E}

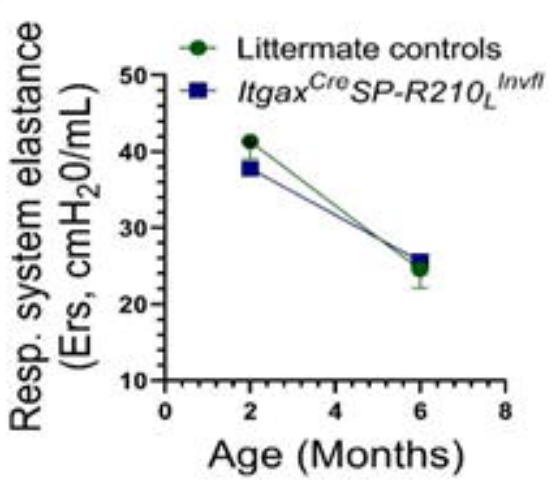

$J$

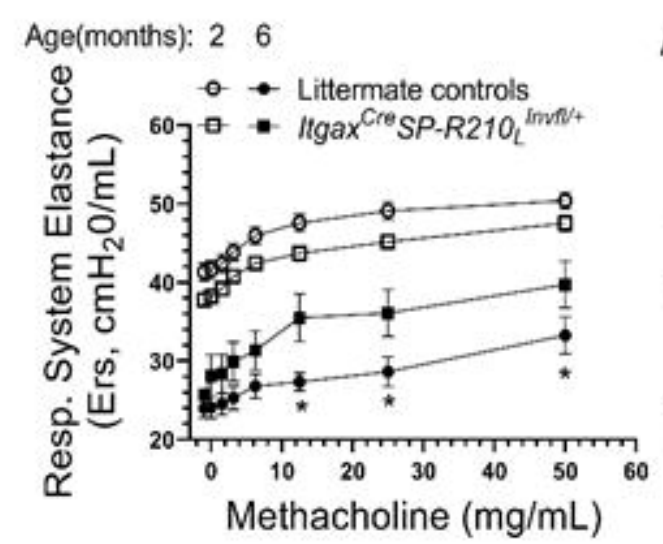

F

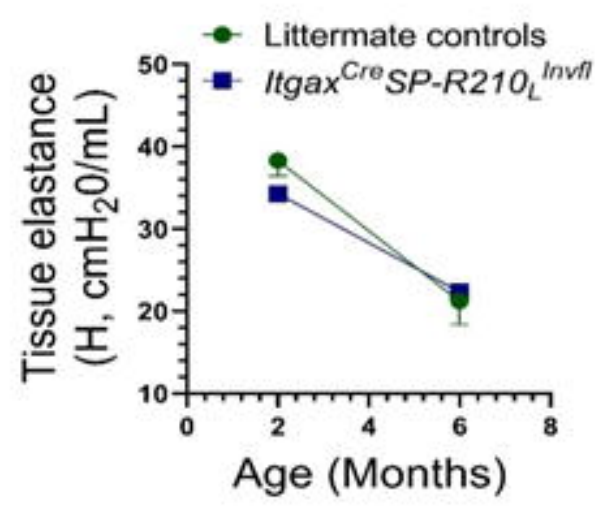

K

Age(months): 26

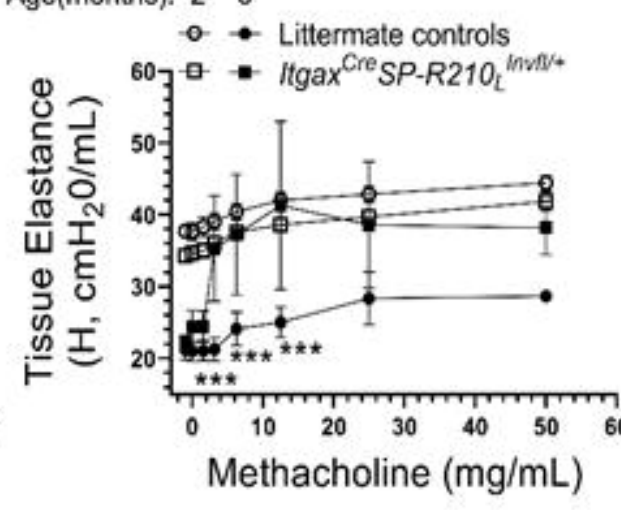

C

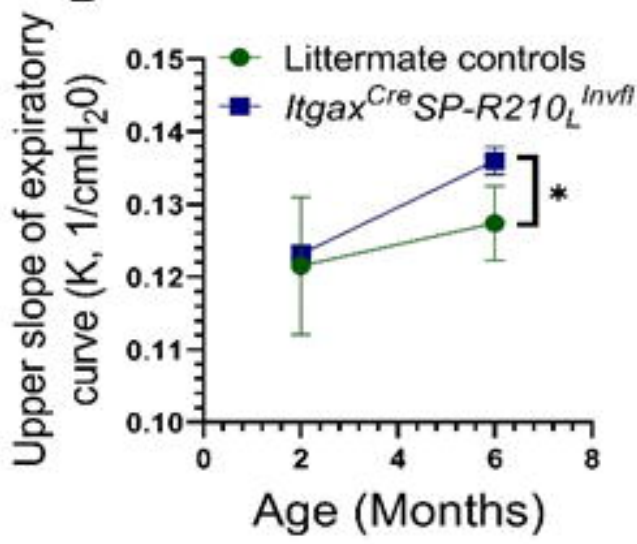

G

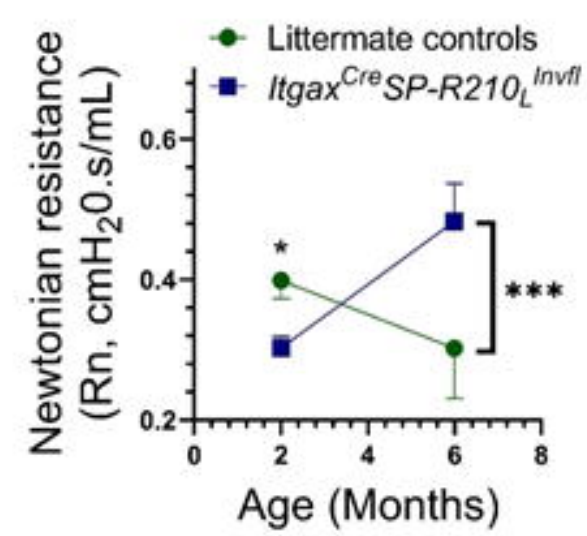

L

Age(months): 26

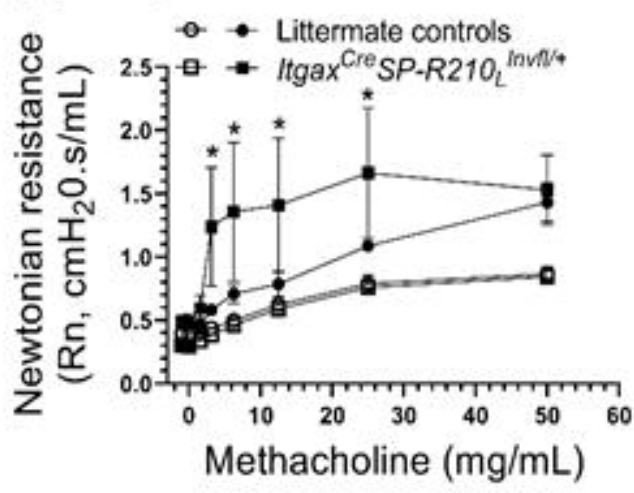

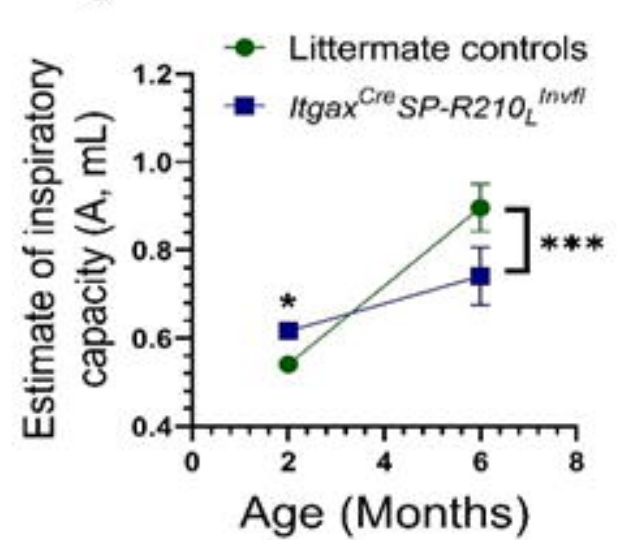

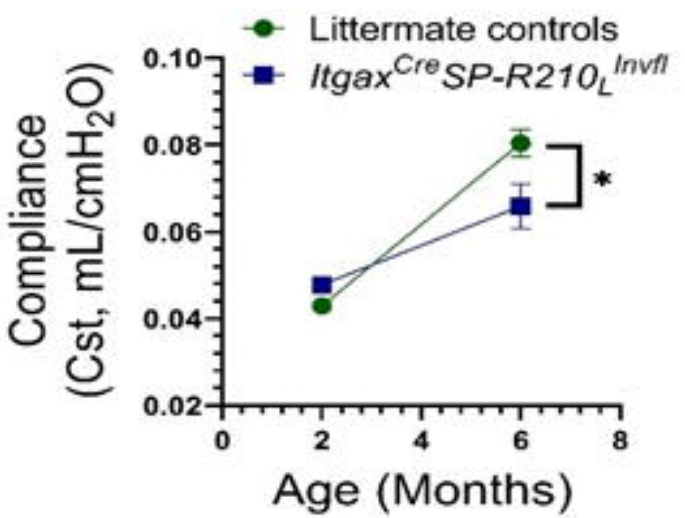

$\mathrm{H}$

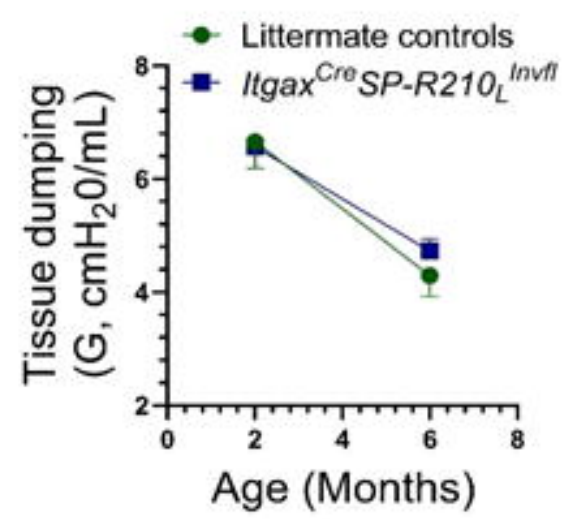

M

Age(months): 26

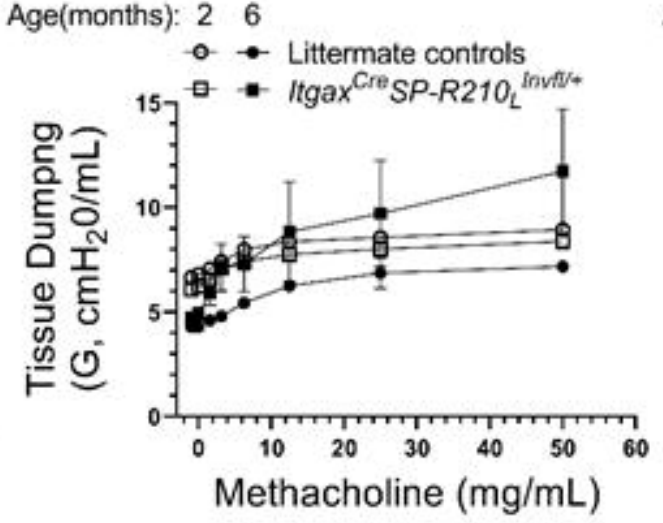

I

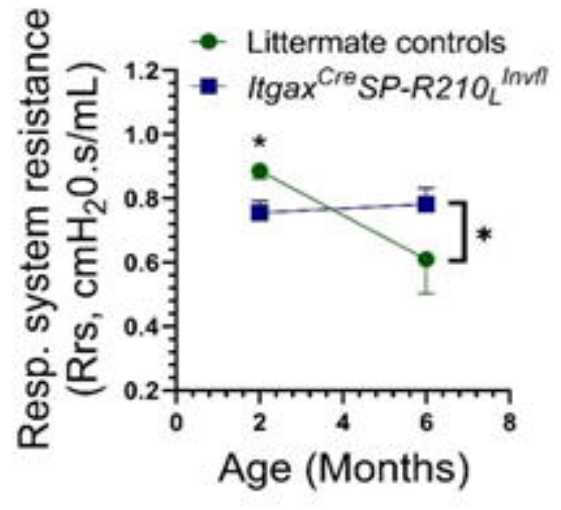

$\mathrm{N}$

Age(months): 26

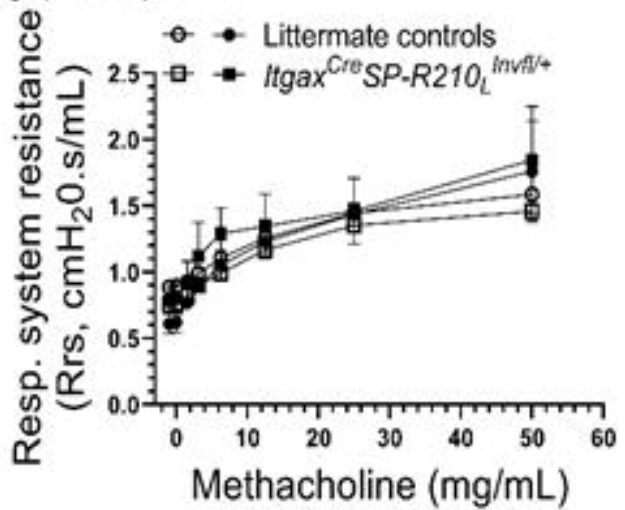

